Nutrition Science en évolution

La revue de l'Ordre professionnel des diététistes du Québec

\title{
Des ressources pour la prise en charge du diabète
}

\section{Jonathan Fontaine et Valérie Savard}

Volume 16, numéro 1, printemps 2018

URI : https://id.erudit.org/iderudit/1048945ar

DOI : https://doi.org/10.7202/1048945ar

Aller au sommaire du numéro

Éditeur(s)

Ordre professionnel des diététistes du Québec

ISSN

2561-620X (numérique)

Découvrir la revue

Citer cet article

Fontaine, J. \& Savard, V. (2018). Des ressources pour la prise en charge du diabète. Nutrition Science en évolution, 16(1), 31-33.

https://doi.org/10.7202/1048945ar d'utilisation que vous pouvez consulter en ligne.

https://apropos.erudit.org/fr/usagers/politique-dutilisation/ 

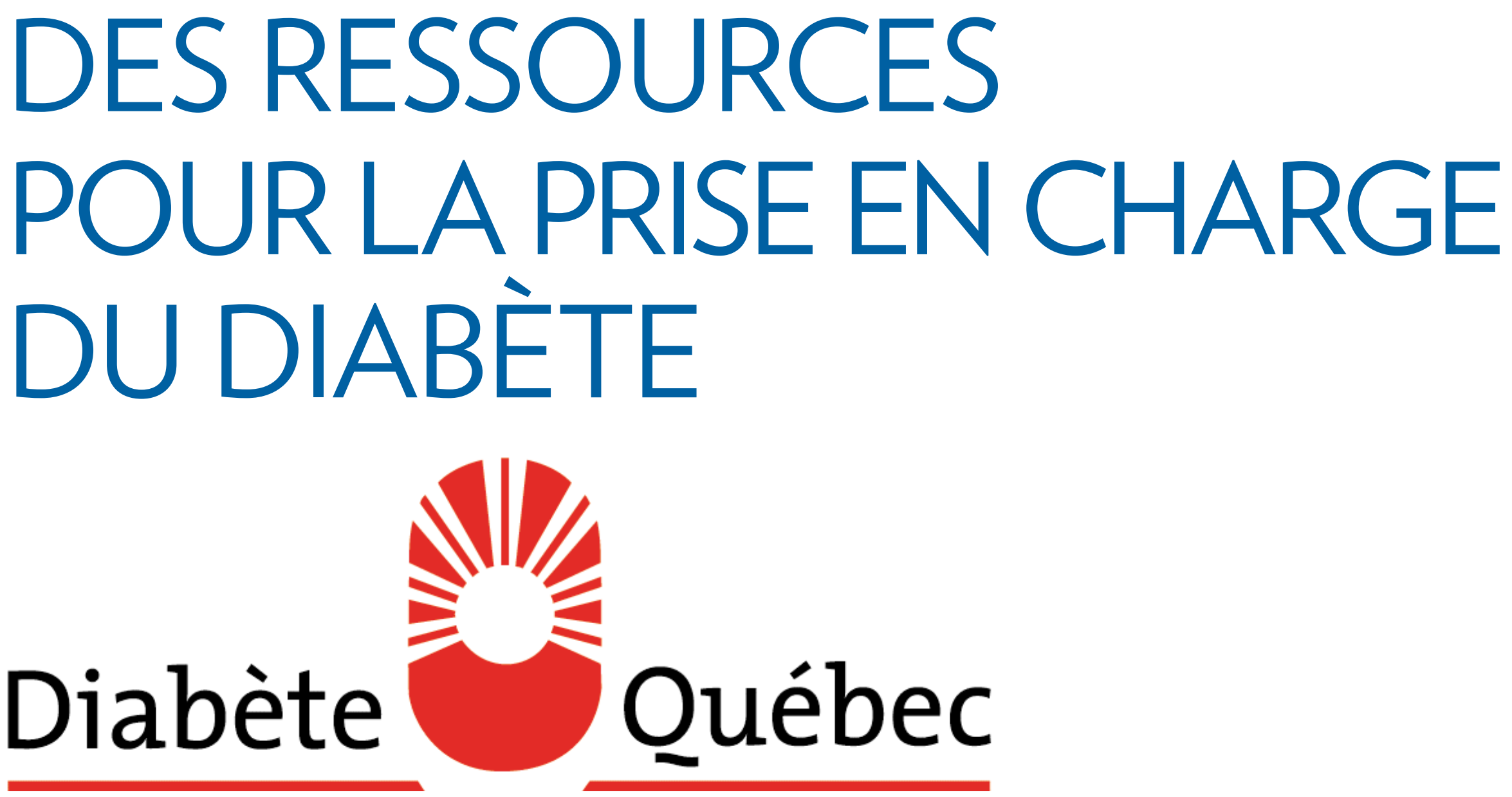

Jonathan Fontaine, Dt.P., gestionnaire principal, Universi-D

Valérie Savard, Dt.P, M. Sc., gestionnaire de projets, Diabète Québec

Selon les plus récentes données de l'Institut national de santé publique du Québec et de Diabète Canada, on compte 880000 personnes diabétiques au Québec, soit plus de $10 \%$ de la population ${ }^{1}$. De ce nombre, 250000 ignorent leur condition ${ }^{2}$. Diabète Québec est une association sans but lucratif au service des personnes diabétiques depuis plus de 60 ans. Elle a pour mission, entre autres, d'améliorer les connaissances et les bonnes pratiques d'enseignement. Diabète Québec sert ainsi de courroie de transmission des connaissances en offrant aux professionnels de la santé des ressources et des outils.

Le rôle des diététistes/nutritionnistes est crucial dans la prise en charge du diabète, notamment pour la préparation et l'enseignement d'un plan d'alimentation personnalisé aux besoins et aux habitudes de chaque personne diabétique. Diabète Québec tient à épauler les diététistes/ nutritionnistes en partageant ses ressources.

\section{Des formations pour bien vous outiller ou garder vos connaissances à jour}

Diabète Québec vient de mettre sur pied l'organisation Universi-D qui chapeaute à présent toutes les activités de formation destinées aux professionnels de la santé, aux personnes diabétiques ou au grand public. Pour en savoir davantage, consultez le www.universi-d.com.

\section{Service d'enseignement Diabétaide}

Les spécialistes du diabète de Diabétaide offrent des programmes d'enseignement destinés aux professionnels de la santé. Le programme Mise à

\footnotetext{
' Données extraites du Système intégré de surveillance des maladies chroniques du Québec (SISMACQ), Institut national de santé publique du Québec (INSPQ), octobre 2017.

${ }^{2}$ Diabète Canada, Rapport sur le diabète 2015 — Agir pour le changement
}

jour sur le diabète couvre les principaux aspects de la gestion du diabète. Il s'adresse particulièrement aux diététistes/nutritionnistes qui débutent avec une clientèle diabétique ou désirent rafraîchir leurs connaissances. Il s'agit d'une formation de deux jours se déroulant au bureau de Diabète Québec, à Montréal. Ces deux journées équivalent à 12 heures de formation reconnue. L'inscription peut se faire en ligne au www.diabete.qc.ca.

\section{Formation en ligne}

Depuis 2015, Diabète Québec offre des cours en ligne grâce à sa boutique de formation. Il s'agit de modules d'autoapprentissage de courte durée sur des thèmes pointus de la gestion du diabète. Au total, 12 formations sont accessibles et plusieurs autres sont à venir. Par exemple, le cours intitulé Activité physique et contrôle glycémique s'adresse aux diététistes/ nutritionnistes qui souhaitent aider leurs patients à bien gérer leur glycémie tout en pratiquant leur sport 
32 ou leurs activités. Nos formations en ligne sont elles aussi reconnues par la SOFEDUC, chaque module de $30 \mathrm{mi}-$ nutes correspond à une demi-heure de formation continue reconnue.

\section{Journées de formation scientifique}

Deux à trois fois par année, Diabète Québec organise des journées de formation scientifique sur le diabète au cours desquelles des chercheurs et des cliniciens viennent présenter les données récentes ou les pratiques à encourager. Comme ces journées répondent aux normes de la SOFEDUC, les participants sont donc admissibles aux unités de formation continue. Les dates des événements sont annoncées sur le site Internet de Diabète Québec.

\section{DES OUTILS UTILES POUR LES PROFESSIONNELS ET LES PATIENTS}

\section{Service InfoDiabète}

Diabète Québec offre un service d'aide aux personnes diabétiques, à leur entourage et aux professionnels de la santé. L'équipe (composée d'une infirmière et de diététistes/nutritionnistes, dont une éducatrice agréée en diabète) répond aux questions sur le diabète par téléphone, par courriel ou par clavardage. Les coordonnées sont les suivantes :

$>$ 514 259-3422 poste 233

$1800361-3504$ poste 233

infodiabete@diabete.qc.ca

www.diabete.qc.ca/fr/dia-

bete-quebec/contact/clavardage

\section{Chaîne YouTube}

Des capsules vidéo ont été enregistrées avec la collaboration de cliniciens et de personnes diabétiques. Elles peuvent, entre autres, être utiles aux personnes diabétiques nouvellement diagnostiquées qui veulent comprendre la maladie ou encore aux personnes qui désirent en apprendre davantage sur divers aspects du diabète. On y trouve de tout, de la gestion d'une hypoglycémie à des programmes d'exercices à faire à la maison en passant par des témoignages de personnes vivant avec le diabète. Elles sont regroupées sur la chaîne YouTube de Diabète Québec: www. youtube.com/user/DiabeteQuebec.

\section{Site Internet}

Au fil du temps, le site www.diabete. qc.ca est devenu une référence de choix sur le diabète dans le monde francophone. On y trouve une grande quantité d'information vulgarisée pour comprendre davantage le diabète et mieux vivre avec la maladie. Plusieurs liens dirigent les visiteurs vers des ressources de qualité. L'information est validée et mise à jour régulièrement. Le site Web est certifié par la fondation Health on the Net qui garantit l'utilité des informations de santé et la conformité aux normes éthiques.

\section{Guide des insulines en version application mobile}

Ce guide a été conçu pour les professionnels de la santé intervenant auprès des personnes diabétiques de type 2 qui doivent commencer un traitement à l'insuline ou en faire l'ajustement. Il uniformise l'approche thérapeutique et l'information transmise aux personnes diabétiques. En le consultant, les nutritionnistes comprendront mieux le mode d'action de chaque type d'insuline et y découvriront toutes les stratégies d'ajustement qui peuvent être envisagées. Le guide est offert sous forme d'application dans les boutiques App Store et Google Play pour les appareils Apple et Android respectivement.

\section{Documents et ouvrages}

Diabète Québec diffuse sur son site Internet des documents (brochures, dépliants, livrets informatifs, etc.) élaborés par ses professionnels de la santé ou provenant d'organisations dans le domaine du diabète. Au besoin, des copies papier peuvent être commandées pour être distribuées à vos patients. Voici les publications les plus demandées :

$>$ Coup d'oeil sur l'alimentation de la personne diabétique: un outil simple pour expliquer les bases de l'alimentation da la personne diabétique.

> Guide avancé du calcul des glucides : un outil d'enseignement du calcul des glucides. Deux versions sont disponibles selon le type de ratio utilisé.

$>$ Guide des produits pour personne diabétique: il recense chaque année les médicaments, lecteurs de glycémie, pompes à insuline et autres produits offerts aux personnes diabétiques sur le marché canadien.

> Dépliants sur les complications: ils décrivent dans un langage simple les principales complications du diabète, comment les traiter et les prévenir. Les sujets abordés comprennent, entre autres, l'insuffisance rénale chronique, la neuropathie, la santé cardiovasculaire et les soins des yeux.

> Symptômes et traitements de l'hypoglycémie et de l'hyperglycémie: ces feuillets peuvent être affichés dans votre milieu de travail ou distribués à vos patients comme aide-mémoire. Au recto, on trouve des illustrations des principaux symptômes de l'hypoglycémie et de l'hyperglycémie. Au verso, les traitements appropriés à chacune des situations sont expliqués en termes clairs.

$>$ Connaitre son diabète pour mieux vivre: rédigé par l'équipe de l'unité de médecine de jour métabolique de l'Hôtel-Dieu du CHUM, ce livre est apprécié par les professionnels autant que par les personnes diabétiques. Il couvre toutes les notions essentielles à la compréhension de la maladie pour favoriser une gestion efficace. 
$>$ Le diabète chez l'enfant et l'adolescent, $2 e$ édition: un livre de référence recommandé aux parents de jeunes diabétiques, mais aussi aux professionnels de la santé et de l'éducation. Il aborde toutes les facettes du diabète en portant une attention particulière aux spécificités liées au jeune âge des personnes atteintes.

Diabète Québec, c'est aussi 40 associations affiliées dans presque toutes les régions du Québec. Elles offrent à leurs membres, majoritairement des personnes diabétiques, des services très variés : réunions d'information, conférences, activités et lignes téléphoniques d'aide. Comme diététistes/ nutritionnistes travaillant avec des personnes diabétiques, vous pouvez les diriger vers l'association de votre région. La liste des associations affiliées se trouve en ligne au www.
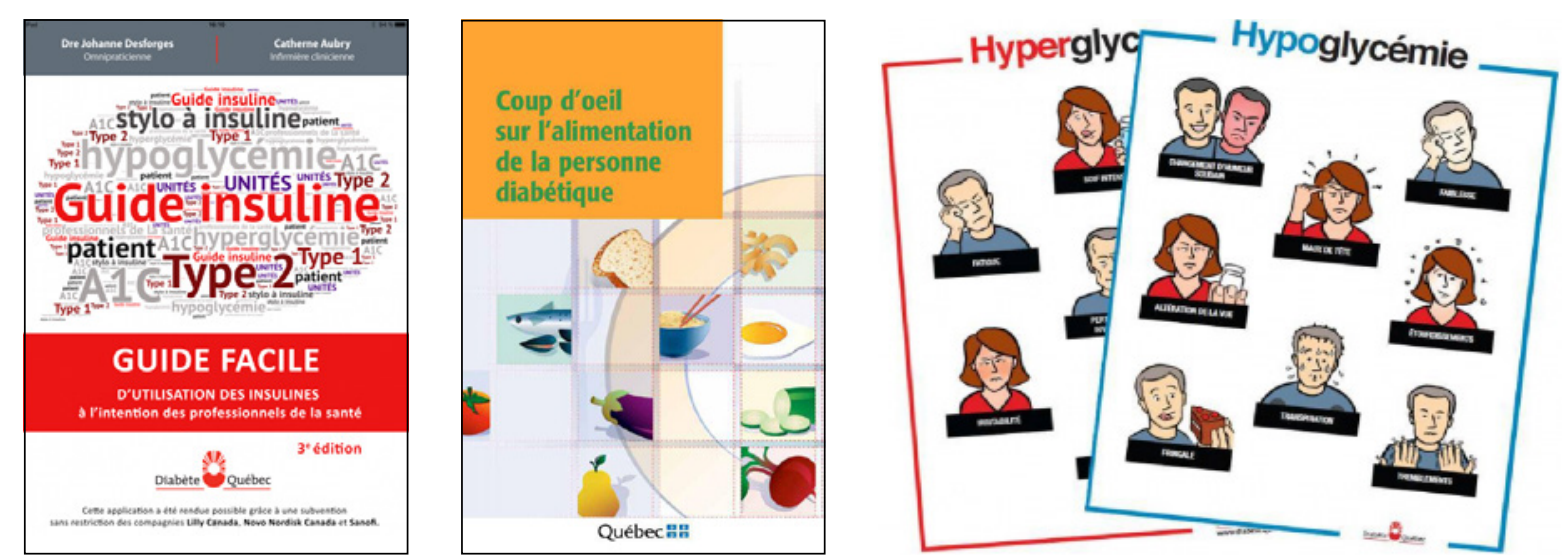

diabete.qc.ca/fr/comprendre-le-diabete/ressources/cartes.

Enfin, les diététistes/nutritionnistes peuvent devenir membres du Conseil professionnel de Diabète Québec (CPDQ). Créé en 1979, le CPDQ regroupe maintenant plus de 1500 professionnels de la santé intervenant dans le domaine du diabète. Le CPDQ a pour rôle de représenter l'expertise en matière de diabète de l'Association. Les professionnels du CPDQ sont par ailleurs informés de toutes les activités proposées par Diabète Québec au moyen d'une infolettre pour les membres.

Certains de ses membres font partie de comités appelés à élaborer des lignes directrices en matière de traitement du diabète et de l'alimentation des personnes diabétiques.

L'adhésion à Diabète Québec ainsi qu'au CPDQ peut se faire en ligne au www.diabete.qc.ca/fr/diabete-quebec/adhesion/pourquoi-devenir-membre.

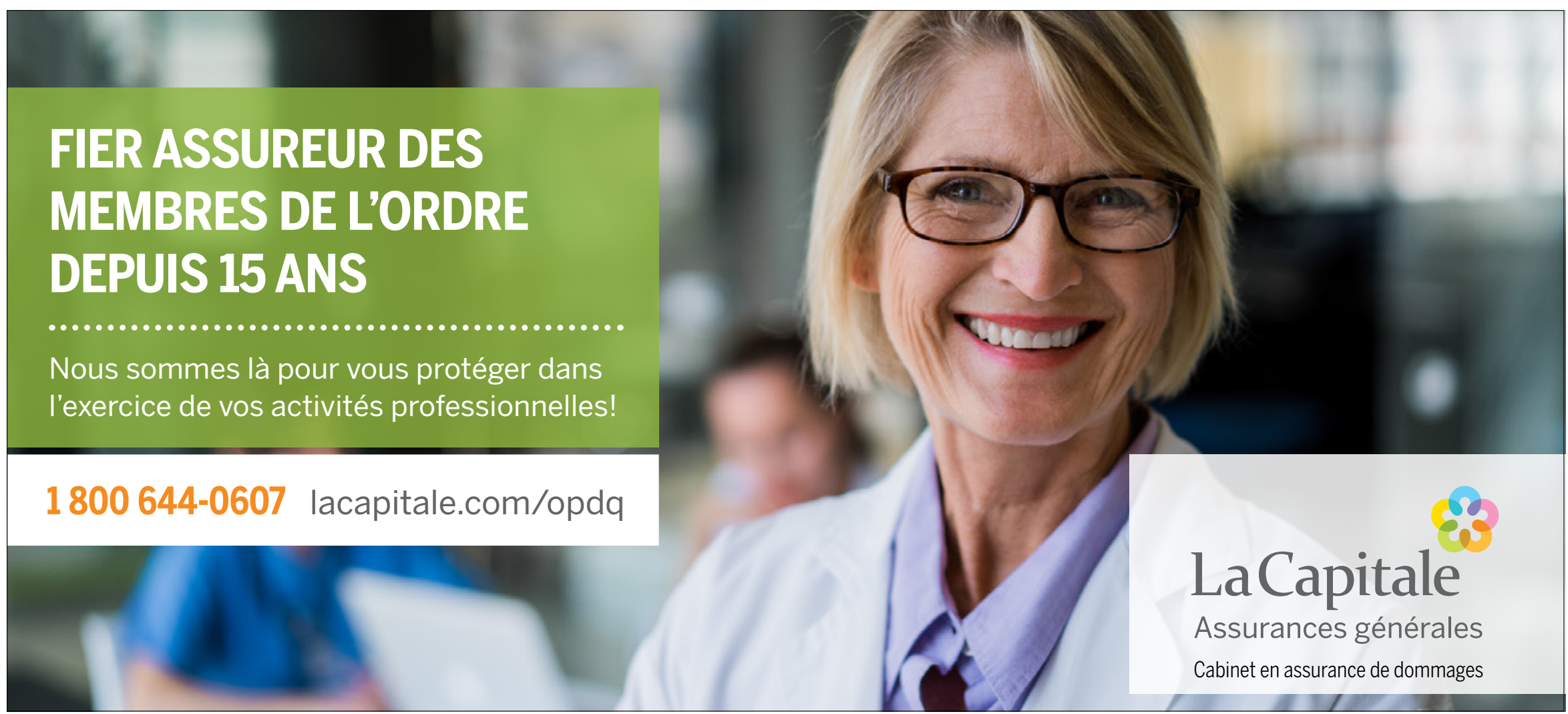

